\title{
Molecular Dynamics Analysis of Coupling between Librational Motions and Isomeric J umps in Chain Molecules
}

\author{
Canan Baysal, Ali R. Atilgan, Burak Erman, and İvet Bahar* \\ Polymer Research Center, School of Engineering, Bogazici University, and TUBITAK \\ Advanced Polymeric Materials Research Center, Bebek 80815, Istanbul, Turkey
}

Received J une 20, 1995; Revised Manuscript Received December 12, $1995^{\circledR}$

\begin{abstract}
The coupling between librational motions and rotational isomeric jumps in polymers is investigated. Librations originate from bond stretching, bond angle distortion, and small-amplitude fluctuations in the bond dihedral angles. Molecular dynamics trajectories for a polymer chain of 100 bonds in vacuo are used for assessing the contribution of librations to the dynamic behavior of chains. Each trajectory is resolved into two sets of components, low and high frequency, representing bond rotameric transitions and librations, respectively. Librational motions are observed to be strongly correlated with the rotational jumps of bonds between isomeric states. In fact, the passage from an isomeric state to another is accompanied by an enhancement in librational motions. Examination of the contribution of librations to the local relaxation behavior of polymers indicates that librations affect the mechanism of motion via two opposing effects. On the one hand, they provide an additional degree of freedom which causes a faster relaxation of the conformational correlation functions associated with the bond dihedral angles. On the other hand, the orientational correlation functions associated with the spatial reorientation of backbone bonds exhibit a slower time decay due to the compensating effect of librational motions. In fact, librational motions are not random but highly directed such that they collectivel y preserve local chain direction and hel $\mathrm{p}$ in accommodating the local reorientations induced by isomeric jumps, which would otherwise result in larger displacements of chain segments. Finally, it is shown that replacing the librations of a trajectory with random, uncorrelated fluctuations leads to an overestimation of the relaxation rate of configurational correlations.
\end{abstract}

\section{Introduction}

Two types of motions dominate local chain dynamics: (i) rotational transitions of backbone bonds from one isomeric state to another and (ii) librational motions including the torsional fluctuations within the energy well of rotameric states, the distortions of bond angles, and the stretching of bonds. The former lies in the lower frequency range of the spectrum, whereas the latter is responsible for the higher frequency motions and constitutes a bath for the former. These two types of motions cooperate during the passage over the energy barriers of the conformational space ${ }^{1}$ and in the relaxation to a new equilibrium state.

One way of quantifying the cooperativity between librational motions and isomeric jumps is to monitor the conformational and orientational motions of chain segments. Conformational motions are those that may be described without reference to an external coordinate system, such as the transitions between trans, gauche $(+)$, and gauche $(-)$ states and the fluctuations in bond dihedral angles. Orientational motions, on the other hand, require a coordinate system for their specification. The angle between a bond at a given time and another bond at a later time is an example. Both conformational and orientational motions are characterized by timedelayed correlation functions (CFs) and the associated correlation times. These are not only predictable by computational methods but also measurable by experiments, such as time-resolved fluorescence or nuclear magnetic resonance. However, the assumptions made in interpreting the experimental results can only be rationalized by computer simulations.

Although there have been several attempts to express the experimental relaxation curves by analytical functions, ${ }^{2}$ most approaches have either neglected the

\footnotetext{
$\otimes$ Abstract published in Advance ACS Abstracts, February 15, 1996.
}

torsional librations ${ }^{3}$ or confined the motion to a few bonds only. ${ }^{4}$ Some studies do not consider threedimensional motions, ${ }^{5}$ and others associate high-frequency effects with additional fast anisotropic processes ${ }^{6}$ incorporating Howarth's treatment of librations. ${ }^{7}$ Derivation of correlation functions by analytical models is rather complex in three dimensions. Cook and $\mathrm{Helfand}{ }^{8}$ developed a one-dimensional analytical model and showed the occurrence of a single-exponential decay rate for conformational correlations, representative of both single and cooperative transitions. A similar model was used by Moro where the coupling between librations and conformational jumps was analyzed on the basis of a linear chain of rotors. ${ }^{9}$ Therein it is demonstrated that librational motions operating after a transition are responsible for attaining the new equilibrium state. Recently Kim and Mattice ${ }^{10}$ took another path in that they investigated the effect of libration on the time evolution of the conformational autocorrelation functions (CACFs) for dihedral angles. Interestingly, they found that the adoption of discrete conformational states excluding librations, as in the basic approach of the dynamic rotational isomeric states (DRIS) theory, ${ }^{11}$ results in an overestimation of conformational correlation times.

In all of the above treatments some questions remain to be answered: How is the time decay of CFs affected by the contribution of librational motions? Do the librations have a random character so that the overall chain dynamics can be adequately predicted by removing them and adopting a DRIS-like treatment? Is the superposition of an additional single-exponential type fast relaxation mechanism on the relaxation spectrum sufficient to account for the effect of librations, as performed in several studies, or do librations act in some cooperative manner and exert different effects on different types of correlations? If some cooperativity exists, i.e., if librations are actively involved in the initiation 
or localization of rotameric jumps, for example, is it possible to retain the effect imposed by librations on large-amplitude movements by simply eliminating the high-frequency components of the overall motion?

In the present study, a molecular dynamics (MD) trajectory is analyzed with the objective of testing the validity of various assumptions made in interpreting experiments and clarifying the above raised issues. Time series obtained from MD simulations are filtered ${ }^{1}$ to obtain smoother trajectories where only low-frequency modes remain. The fluctuations in bond lengths and angles and/or dihedral angles are also turned off by using an operator formalism, and the consequences of eliminating these degrees of freedom on the relaxation mechanism are explored. Alternatively, these degrees of freedom are assigned Gaussianly distributed random variables about their equilibrium values. CFs calculated by using these modified trajectories are compared to those determined from the original MD, in order to assess the effects of different levels of approximation.

In the next section, the two types of correlation functions considered in the present analysis are introduced as well as the methods adopted in interpreting the results. Next, the time decay of correlation functions obtained after subjecting the original MD trajectory to various operations is presented, demonstrating the strong coupling between low- and high-frequency motions and the nontrivial dependence of the local relaxation behavior on this coupling.

\section{Method and Calculations}

Correlation Functions. The effect of librations on the local relaxation phenomenon will be explored on the basis of two types of autocorrelation functions: (i) the orientational correlation functions (OACFs) associated with the reorientation of backbone bonds. This is expressed in terms of the instantaneous unit vector $\mathbf{I}_{\mathrm{i}}(\tau)$ along the ith bond as

$$
M[I(\tau)]=\frac{1}{2}\left\{3 \overline{\left\langle\left[\mathbf{I}_{\mathbf{i}}(\mathrm{t}) \cdot \mathbf{I}_{\mathrm{i}}(\mathrm{t}+\tau)\right]^{2}\right\rangle}-1\right\}
$$

(ii) the second CACF describing the time evolution of the rotational isomeric states of backbone bonds. This is given by

$$
\mathrm{M}[\varphi(\tau)]=\frac{1}{2}\left\{3 \overline{\left.\cos ^{2}\left[\varphi_{\mathrm{i}}(\mathrm{t}+\tau)-\varphi_{\mathrm{i}}(\mathrm{t})\right]\right\rangle}-1\right\}
$$

where $\varphi_{\mathrm{i}}(\mathrm{t})$ is the dihedral angle of the ith bond at timet. The broken brackets in eqs 1 and 2 refer to the ensemble average over internal bonds, and the overbar denotes the time average over all conformational transitions starting at various $t$ and occurring within the time interval $\tau$. Here, the section $30 \leq \mathrm{i}$ $\leq 70$ will be considered for a chain of $n=100$ bonds.

Molecular Dynamics Method. MD simulations are carried out for a united atom model polyethylene (PE) chain of $n$ $=100 \mathrm{CH}_{2}$ units. Units are indexed from I to $\mathrm{n}$. The backbone bond $\mathrm{I}_{i}$ connects the $\mathrm{CH}_{2}$ groups $\mathrm{i}-1$ and $\mathrm{i}$. The time-evolved coordinates of atoms are obtained as an array of position vectors $\mathbf{r}_{\mathrm{i}}(\mathrm{t})=\left[\mathrm{x}_{\mathrm{i}}(\mathrm{t}) \mathrm{y}_{\mathrm{i}}(\mathrm{t}) \mathrm{z}_{\mathrm{i}}(\mathrm{t})\right]^{\top}, 0 \leq \mathrm{i} \leq \mathrm{n}$, where the superscript $T$ denotes the transpose. These are transformed into a set of general ized coordinates, consisting of (i) bond dihedral angles $\varphi_{\mathrm{i}}, 2 \leq \mathrm{i} \leq \mathrm{n}-1$, (ii) bond angles, $\theta_{\mathrm{i}}, 1 \leq \mathrm{i} \leq \mathrm{n}-1$, (iii) bond lengths, $\mathrm{l}_{\mathrm{i}}, \mathrm{l} \leq \mathrm{i} \leq \mathrm{n}$, of the particles at any instant in time, following the formulation previously presented. ${ }^{12}$ Here the time arguments are omitted for brevity. For convenience, we will denote as $\mathbf{Q}_{1}$ the transformation matrix or operator that permits the passage from the set of position vectors to that of the generalized coordinates, i.e.

$$
\mathbf{Q}_{1}:\left[\begin{array}{l}
x_{i} \\
y_{i} \\
z_{i}
\end{array}\right] \rightarrow\left[\begin{array}{l}
l_{i} \\
\theta_{i} \\
\varphi_{\mathrm{i}}
\end{array}\right]
$$

The inverse operator, $\mathbf{Q}_{1}^{-1}$, transforms from the generalized coordinates $\left[\mathrm{I}_{i}, \theta_{\mathrm{i}}, \varphi_{\mathrm{i}}\right]^{\top}$ to the set of position vectors, $\left[\mathrm{x}_{i}, \mathrm{y}_{\mathrm{i}}, \mathrm{z}_{\mathrm{i}}\right]^{\top}$. The set of generalized coordinates, obtained for all units in a sequence of 40 bonds located in the middle of the chain and recorded at $4 \mathrm{fs}$ intervals, will be referred to as the original trajectory. The latter will be subjected to various operations as will be presented below.

The chain is chosen to be in vacuo so that only those interactions generated by the polymer itself are operative. The overall potential is the sum of (i) a harmonic bond stretching and (ii) a harmonic bond bending function, (iii) a quintic function that accounts for the intrinsic torsional potential of dihedral angles and first-order interactions between chain atoms separated by three bonds, and (iv) a Lennard-J ones potential, which operates on atom pairs that are separated by four or more bonds and are within $12 \AA$ distance of each other. Further details of the simulation method are provided in our previous work.1,12,13 Initially, a 25 ps equilibration time is allowed. The total simulation duration after equilibration is $\sim 260 \mathrm{ps}$. The temperature is kept constant at $300 \mathrm{~K}$.

Filtering Technique. The contribution of modes with particular frequency ranges $v_{\min } \leq v \leq v_{\max }$ may be extracted from the original MD trajectories by applying the following filtering method: ${ }^{14}(\mathrm{a})$ The original trajectory $A(t)$ is Fourier transformed into the frequency domain,

$$
\hat{A}(v)=\int_{-\infty}^{\infty} A(t) e^{-j v t} d t
$$

(b) A filter function $\mathrm{F}(v)$ that extracts the frequency components within the range of interest is applied, as

$$
\begin{gathered}
\hat{\mathrm{A}}^{\prime}(v)=\hat{\mathrm{A}}(v) \mathrm{F}(v) \\
\mathrm{F}(v)=\left\{\begin{array}{l}
1 ; v_{\text {min }}<v<v_{\text {max }} \\
0 ; v<v_{\text {min }}, v>v_{\text {max }}
\end{array}\right.
\end{gathered}
$$

(c) $\hat{\mathrm{A}}^{\prime}(v)$ is inverse Fourier transformed to the time domain and the frequency-filtered trajectory $\mathrm{A}^{\prime}\left(v_{\min }<v<v_{\max }, \mathrm{t}\right)$ is obtained as,

$$
\mathrm{A}^{\prime}\left(v_{\text {min }}<v<v_{\max }, \mathrm{t}\right)=\int_{-\infty}^{\infty} \hat{\mathrm{A}}^{\prime}(v) \mathrm{e}^{\mathrm{j} v \mathrm{t}} \mathrm{d} v
$$

In a recent MD study with the same model chains, it was shown that the modes responsible for isomeric transitions are those having frequencies below $20 \mathrm{~cm}^{-1}$, approximately, whereas the overall relaxation spectrum contains frequencies up to $\sim 4000 \mathrm{~cm}^{-1}$. Retaining these low-frequency modes is termed low-pass filtering below $20 \mathrm{~cm}^{-1}$. The cutoff value of $20 \mathrm{~cm}^{-1}$ is not definitive, however, and lower or higher values may be chosen depending on the precision required by the quantity of interest.

Reconstruction of Trajectories Subject to Various Operations. The original MD trajectories are subjected to the following operations to obtain the so-called modified trajectories: (a) The fluctuations in bond lengths and bond angles are removed from the trajectories by replacing these variables with their mean values. This may be expressed by the operation

$$
\mathbf{Q}_{2}:\left[\begin{array}{c}
\bar{l}_{\mathrm{i}}+\Delta \mathrm{l}_{\mathrm{i}} \\
\bar{\theta}_{\mathrm{i}}+\Delta \theta_{\mathrm{i}} \\
\varphi_{\mathrm{i}}
\end{array}\right] \rightarrow\left[\begin{array}{c}
\bar{l}_{\mathrm{i}} \\
\bar{\theta}_{\mathrm{i}} \\
\varphi_{\mathrm{i}}
\end{array}\right]
$$

where the instantaneous bond lengths and bond angles are expressed as a sum of their mean values and their fluctuations, as

$$
\begin{gathered}
\mathrm{I}_{\mathrm{i}}=\overline{\mathrm{I}}_{\mathrm{i}}+\Delta \mathrm{l}_{\mathrm{i}} \\
\theta_{\mathrm{i}}=\bar{\theta}_{\mathrm{i}}+\Delta \theta_{\mathrm{i}}
\end{gathered}
$$


Table 1. Characteristics of the Trajectories Utilized for Extracting OACFs and Their Orientational Correlation Times ${ }^{\mathrm{a}}$

\begin{tabular}{clcc}
\hline OACF & operation & $\tau_{\mathrm{c}}(\mathrm{ps})$ & $\tau_{\mathrm{d}} \tau_{\mathrm{co}}$ \\
\hline $\mathrm{i}$ & none & 7.81 & 1.00 \\
$\mathrm{ii}$ & filtered $^{\mathrm{b}}$ & 9.39 & 1.20 \\
$\mathrm{iii}$ & $\mathrm{Q}_{1}{ }^{-1} \mathrm{O}_{2} \mathrm{Q}_{1}$ & 5.62 & 0.72 \\
iv & $\mathrm{Q}_{1}{ }^{-1} \mathrm{Q}_{3} \mathrm{Q}_{1}$ & 4.09 & 0.52 \\
v & $\mathrm{Q}_{1}{ }^{-1} \mathrm{Q}_{4} \mathrm{Q}_{1}$ & 5.51 & 0.71 \\
vi & $\mathrm{Q}_{1}{ }^{-1} \mathrm{Q}_{5} \mathrm{Q}_{1}$ & 3.59 & 0.46
\end{tabular}

${ }^{\text {a }} \tau_{\mathrm{c}}$ : orientational correlation times. $\tau_{\mathrm{co}}: \tau_{\mathrm{c}}$ of original MD run. b Using a low-pass filter of $20 \mathrm{~cm}^{-1}$.

The mean bond lengths and bond angles are $1.53 \AA$ and $109.5^{\circ}$, for all bonds. Comparison of the modified trajectory with the original trajectories will provide information on the contribution of the librations in bond lengths and bond angles on the local chain relaxation mechanism. Clearly the modified set of position vectors may be generated by the application of the inverse operator $\mathbf{Q}_{1}^{-1}$ on the modified generalized coordinates obtained in eq 7. In summary, a modified set of position vectors is obtained by premultiplication of the original array by $\mathbf{Q}_{1}^{-1} \mathbf{Q}_{2} \mathbf{Q}_{1}$.

(b) In addition to bond length and bond angles, torsional librations about rotational isomeric minima may be eliminated by replacing the original trajectories by a new one modified according to the operation

$$
\mathbf{Q}_{3}:\left[\begin{array}{l}
\bar{l}_{\mathrm{i}}+\Delta \mathrm{I}_{\mathrm{i}} \\
\bar{\theta}_{\mathrm{i}}+\Delta \theta_{\mathrm{i}} \\
\bar{\varphi}_{\mathrm{i}}+\Delta \varphi_{\mathrm{i}}
\end{array}\right] \rightarrow\left[\begin{array}{c}
\bar{l}_{\mathrm{i}} \\
\bar{\theta}_{\mathrm{i}} \\
\bar{\varphi}_{\mathrm{i}}
\end{array}\right]
$$

Here $\bar{\varphi}_{i}$ represents the rotational isomeric state values assumed by the ith dihedral angle. This is given by a step function of the form ${ }^{15}$

$$
\bar{\varphi}_{\mathrm{i}}(\tau)=\left\{\begin{array}{cr}
0^{\circ} & -60^{\circ} \leq \varphi_{\mathrm{i}}(\tau) \leq 60^{\circ} \\
120^{\circ} & \varphi_{\mathrm{i}}(\tau) \geq 60^{\circ} \\
-120^{\circ} & \varphi_{\mathrm{i}}(\tau) \leq-60^{\circ}
\end{array}\right.
$$

The values $0^{\circ},-120^{\circ}$, and $+120^{\circ}$ correspond to the respective isomeric states trans, gauche $(-)$, and gauche $(+)$.

(c) Alternatively, normal fluctuations may be superimposed on bond lengths and bond angles, which would provide insights into the differences between random librations and those occurring in the original MD trajectories. Accordingly, the instantaneous bond lengths and bond angles are expressed as Gaussianly distributed random variables with the mean values $1.53 \AA$ and $109.5^{\circ}$ and covariances $0.10 \AA$ and $10^{\circ}$, respectively. This transformation may be written as

$$
\mathbf{Q}_{4}:\left[\begin{array}{c}
\overline{\mathrm{T}}_{\mathrm{i}}+\Delta \mathrm{l}_{\mathrm{i}} \\
\bar{\theta}_{\mathrm{i}}+\Delta \theta_{\mathrm{i}} \\
\varphi_{\mathrm{i}}
\end{array}\right] \rightarrow\left[\begin{array}{c}
\overline{\mathrm{T}}_{\mathrm{i}}+\Delta \mathrm{l}_{\mathrm{i}}^{\mathrm{n}} \\
\bar{\theta}_{\mathrm{i}}+\Delta \theta_{\mathrm{i}}^{\mathrm{n}} \\
\varphi_{\mathrm{i}}
\end{array}\right]
$$

Here the superscripts $n$ refer to the normal distribution, and the dihedral angles are left unchanged.

(d) A final transformation to be considered in the calculations is $\mathbf{Q}_{5}$, represented as

$$
\mathbf{Q}_{5}:\left[\begin{array}{c}
\overline{\mathrm{T}}_{\mathrm{i}}+\Delta \mathrm{I}_{\mathrm{i}} \\
\bar{\theta}_{\mathrm{i}}+\Delta \theta_{\mathrm{i}} \\
\varphi_{\mathrm{i}}
\end{array}\right] \rightarrow\left[\begin{array}{c}
\overline{\mathrm{i}}_{\mathrm{i}}+\Delta \mathrm{I}_{\mathrm{i}}{ }^{\mathrm{n}} \\
\bar{\theta}_{\mathrm{i}}+\Delta \theta_{\mathrm{i}}{ }^{\mathrm{n}} \\
\bar{\varphi}_{\mathrm{i}}
\end{array}\right]
$$

which is identical to $\mathbf{Q}_{4}$, except for the replacement of the original torsional angles by their isomeric state values following eq 10. Clearly, in all cases a-d the modified set of position vectors is constructed by application of the operation $\mathbf{Q}_{1}^{-1} \mathbf{Q}_{\mathrm{m}}$ $\mathbf{Q}_{1}$, with $\mathrm{m}=2-5$, on the original position vectors.

Table 1 provides a summary of the operations adopted in obtaining modified trajectories subject to various approximations, starting from the original MD trajectory. It should be

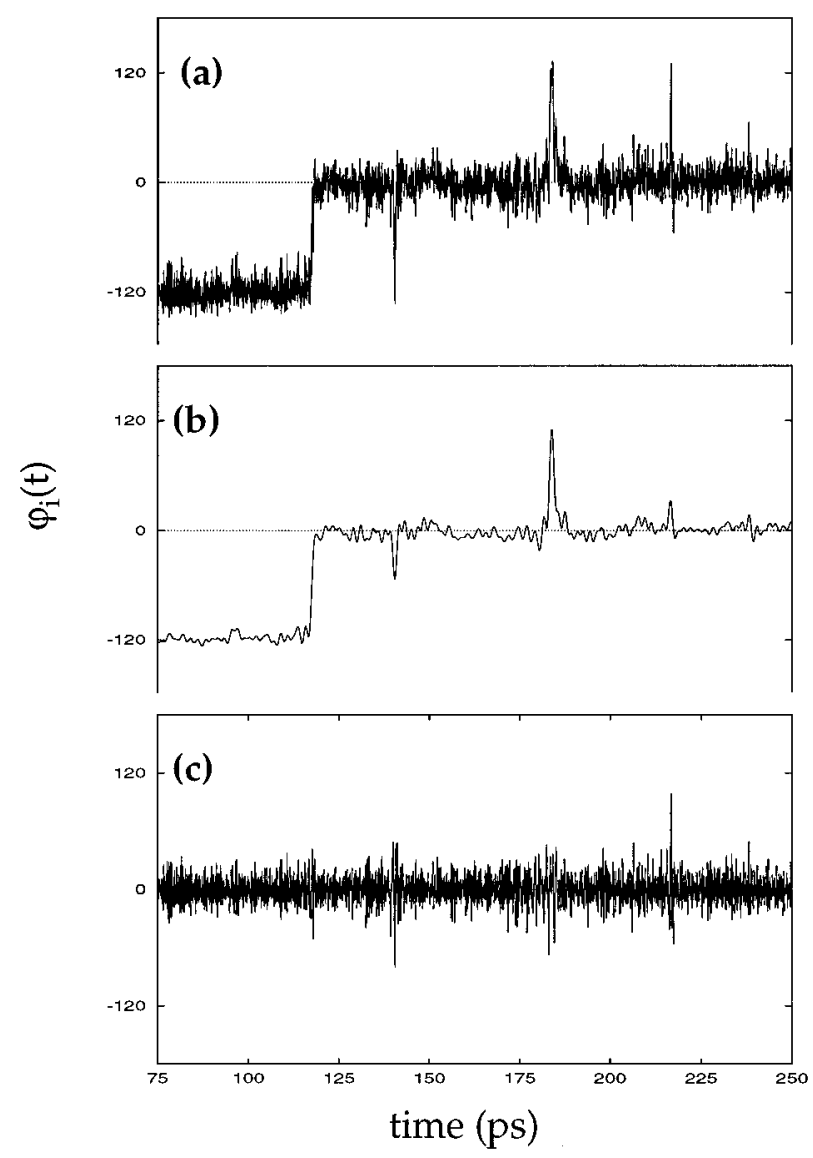

Figure 1. Portion of a representative dihedral angle trajectory obtained from MD simulations: (a) trajectory containing all frequencies, (b) same trajectory containing frequencies below $20 \mathrm{~cm}^{-1}$, and (c) same trajectory with frequencies above 20 $\mathrm{cm}^{-1}$. The excited fluctuations in part $c$ are synchronized with the rotational isomeric transitions displayed in parts $a$ and $b$, which demonstrate the coupling between low- and highfrequency modes.

noted that the perturbations applied to the various degrees of freedom do not result from a change in the potential field used in MD simulations. To the contrary, all trajectories originate from the same MD run with a given realistic potential field. In the earlier work of Helfand et al. ${ }^{16}$ the force constants in the potentials were changed so as to constrain $l_{i}$ and $\theta_{i}$. This is a different approach which can provide an estimation of the effect of constraining the librational motions on the relaxation mechanism. The present approach, on the other hand, provides a direct measure of the perturbation of the MD trajectories by several factors, including the elimination of a class of operating modes via filtering technique, the removal of the librational motions, which may be directed, random, or both, and the imposition of a white noise for approximating the additional mobility imparted by the high-frequency librational motions.

\section{Results}

Coupling between Low- and High-Frequency Modes. For illustrative purposes, a 250 ps portion of the trajectory for one of the dihedral angles is presented in Figure 1a. The same trajectory, subjected to a lowpass filtering procedure truncating all modes with frequencies above $20 \mathrm{~cm}^{-1}$, is shown in Figure $1 \mathrm{~b}$. Figure 1c shows the trajectory containing frequencies above $20 \mathrm{~cm}^{-1}$ only. Comparison of the curves in Figures $1 a$ and $b$ shows that motions associated with isomeric transitions are well represented by the 0-20 $\mathrm{cm}^{-1}$ range of the frequency spectrum. It is interesting to note that the bond rotations in curve $a$ or $b$ are 


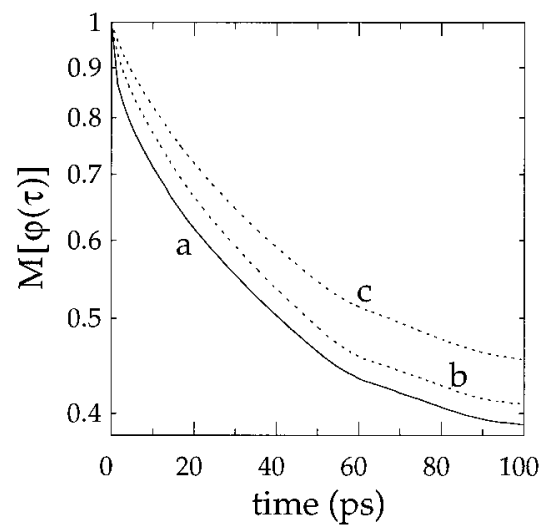

Figure 2. Time decay of CACFs obtained from (a) the original dihedral angle MD trajectories, (b) low-pass filtered trajectories bel ow $20 \mathrm{~cm}^{-1}$ using eqs 4-6, and (c) regenerated trajectories where only equilibrium values of the dihedral angles are all owed, conforming with eq 10. Logarithmic scale is used in the ordinate for clarity.

accompanied by an enhancement in the torsional fluctuations shown in part c. The occurrence of such excited librations, synchronous with bond rotational transitions, is direct evidence of the correlations between fast and slow motions and, in particular, of their enhanced coupling during rotational isomerization.

Conformational Autocorrelations. The CACFs, $\mathrm{M}[\varphi(\tau)]$, evaluated using different approaches are presented in Figure 2, up to $\tau=100 \mathrm{ps}$. Curve a represents the result from the original MD trajectory. Curve $b$ is obtained by applying a low-pass filtering bel ow $20 \mathrm{~cm}^{-1}$. Curve c, on the other hand, is obtained by subjecting the original generalized coordinates to the transformation operator $\mathbf{Q}_{3}$. This eliminates all bond angle, bond length, and torsional librations. However, eliminating the bond lengths and bond angles is inconsequential since the CACF is a quantity that depends purely on dihedral angles. In this approximation, the conformational dynamics corresponds to that adopted in the DRIS model. This curve also compares to that given by Kim and Mattice. ${ }^{10}$ It is evident from the figure that both approximations $b$ and c predict a slower decay compared to the original trajectory (a). The CACF from the filtered trajectory, curve $b$, indicates that the departure between the approximated and original behavior arises primarily from a fast decay to $\sim 0.86$ of the ordinate value in curve a within tenths of picoseconds, which is absent in curve b. This initial decay in curve $a$ is clearly induced by the high-frequency motions which have been eliminated in the filtered trajectory (b). The departure from the original CACF is even stronger in curve $c$, where all torsional librations are extinguished following eqs $4-6$.

A comparison al ong the same lines was made by $\mathrm{Kim}$ and Mattice for polybutadiene chains in the bulk state and a similar result was obtained. ${ }^{10}$ Therein, it was argued that the dynamics is governed by two relaxation phenomena at separate time scales: one is responsible for librations and the other for conformational transitions. Thus, neglect of librational motions leads to slower relaxation insofar as the CACF is concerned. This approximation can be partly overcome by rescaling of the decay curves at short times. This explains the good agreement between the CACF s extracted from Brownian dynamics simulations and those predicted by the DRIS formalism in which librational motions are not accounted for. ${ }^{13}$

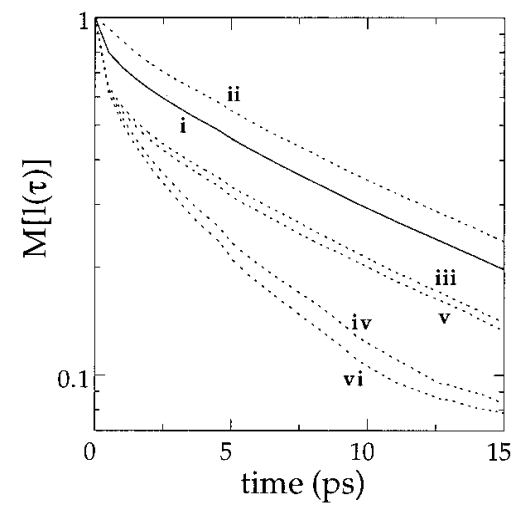

Figure 3. Time decay of bond OACFs obtained with the various levels of approximation listed in Table 1 . The solid curve, labeled i, refers to the original trajectory. Curve ii is the low-pass filtered trajectory. Curves iii-vi result from different constraints applied on bond bending, stretching, and torsion degrees of freedom, as outlined in Table 1. Logarithmic scale is used in the ordinate for clarity.

A mere analysis of the decays of CACF provides an incomplete picture of the relaxation mechanism. A more thorough understanding of the role of highfrequency motions in local dynamics is gained by examining the time-dependent orientation of chain segments in space, in the presence and absence of librations.

Orientational Autocorrelations. The OACFs of bond vectors, $M[I(\tau)]$, obtained by subjecting a given $M D$ trajectory to different operations, are presented in Figure 3, up to $\tau=15$ ps. Note that only the shorttime range is given to emphasize the differences between the initial decay of the curves. They all decay to zero eventually. Curve i represents the correlation function given by the original MD trajectory. The curves labeled ii-vi result from the trajectories modified following the procedures summarized in Table 1. Mainly, curve ii refers to the low-pass $\left(20 \mathrm{~cm}^{-1}\right)$ filtered trajectory. Curves iii and iv result from the application of the operators $\mathbf{Q}_{1}^{-1} \mathbf{Q}_{2} \mathbf{Q}_{1}$ and $\mathbf{Q}_{1}^{-1} \mathbf{Q}_{3} \mathbf{Q}_{1}$, respectively. This allows the extraction of the contribution of librational motions to the observed OACFs. Cases $v$ and vi follow from the operations $\mathbf{Q}_{1}^{-1} \mathbf{Q}_{4} \mathbf{Q}_{1}$ and $\mathbf{Q}_{1}^{-1} \mathbf{Q}_{5} \mathbf{Q}_{1}$ applied to the set of position vectors recorded in the original MD trajectories. Here bond lengths and bond angles are assigned Gaussianly distributed random fluctuations, which permit the visualization of the difference between random librations and those cooperatively operating in the original MD run. The orientational correlation times $\tau_{\mathrm{c}}$ and their ratios $\tau_{\mathrm{d}} \tau_{\mathrm{co}}$ with respect to that resulting from the original MD run, $\tau_{\mathrm{co}}$, are listed in the last two columns of Table 1 in order to provide a measure of the effect of the various operations on the apparent OACFs.

The low-pass filtered trajectory yiel ds a slower decay of the OACF than that of the original trajectory, as the comparison of the curves i (solid) and ii reveals. The more interesting feature observed in Figure 3 is that all other curves, iii-vi, exhibit faster decays compared to the original curvei. Even the supression of librations in bond lengths and bond angles, which is represented by curve iii, enhances the decay rate of $M[I(\tau)]$ by a considerable amount. A decrease in orientational correlation time of $28 \%$ is induced by eliminating the vibrations in bond lengths and bond angles.

In many experimental and computational studies it is assumed that librations, especially those associated with stretching and bending, have a random charac- 
ter.6,7 Curves $v$ and vi in Figure 3 result from the imposition of Gaussianly distributed random fluctuations on the equilibrium values of bond lengths and angles. Examination of these curves demonstrates that the replacement of the original directed librational motions by random fluctuations leads to a significantly faster loss of orientation. The relaxation time is de creased by a factor of 0.71 and 0.46 , respectively, in curves $\mathrm{v}$ and $\mathrm{vi}$, compared to the original behavior. These results invite attention to the inadequacy of interpreting the stretching, bending, and torsional librational motions as a random accelerating relaxation mechanism.

\section{Interpretation of Results and Discussion}

Our recent molecular dynamics study of chain dynamics indicates that librations have components that are strongly correlated with slower rotameric transitions. ${ }^{1}$ The basic feature of this coupling is that two or more degrees of freedom may librate in a concerted fashion to localize a large-scale rotameric transition in space which would otherwise result in excessive translations of points along the chain. An inspection of the three trajectories given in Figure 1 gives a good representation of the coupling between librations and rotational isomeric jumps. A definite coupling between bond rotational isomeric jumps and librational motions is evidenced by the enhanced activity of the high-frequency modes (Figure 1c) during passage over rotational energy barriers. In addition to such coupled librations, there are also librations in various degrees of freedom which are random and not correlated in the manner stated.

Removal of frequencies higher than $20 \mathrm{~cm}^{-1}$ from the trajectory of Figure 1 eliminates most of the librations that are coupled to, or correlated with, rotameric transitions as well as those that are uncoupled. This results in the slowing down of the relaxation of conformational and configurational transitions shown by curve $b$ in Figure 2 and curve ii in Figure 3. This is essentially due to the elimination of some pathways of relaxation from the trajectory which otherwise would lead to faster decay of correlations.

Operating on the trajectory with $\mathbf{Q}_{2}$ eliminates all librations of bond lengths and angles and therefore excludes the possible localization effects that would result from the concerted motions of these degrees of freedom. Consequently, any change in a torsional angle of a given bond has to move the remaining parts of the chain as a rigid body, which induces large displacements and leads to a rapid decay of the OACF shown in Figure 3, curve iii.

Likewise, applying the operator $\mathbf{Q}_{3}$ on the trajectory eliminates all localization effects resulting from librations in torsion angles as well as from those in bond angles and bond lengths. In the absence of such cooperative fluctuations, a jump in a torsion angle from one isomeric minimum to another would induce largescale displacements of the tails flanking the rotating bond. Such large changes in orientations result in the rapid decay of the OACF shown in curve iv. On the other hand, removal of librations by the application of $\mathbf{Q}_{3}$ does not accelerate the loss of conformational correlations: A slowing down in the decay of the CACF (Figure 2c) results because of the removal of the rapid pathways of relaxation. It should be noted that CACFs, and in particular that of Hall and Helfand, have been commonly used in literature for interpreting or fitting the time decay of OACFs, without a rational justification. The present study clearly demonstrates that the responses of OACF s and CACF s to librations differ from each other, and consequently, the use of the same functional form to express both correlation functions may not be adequate.

It is to be noted that the replacement of the original librations with uncorrelated ones results in OACF curves (curves $v$ and vi in Figure 3 ) that are significantly below the OACF curve of the original trajectory. In fact, the new OACF curves lie very close to the corresponding curves iii and iv obtained in the absence of librations. It may thus be concluded that random librations are far from representing the effects of the coordinated librations and may not be adopted as appropriate mechanisms of relaxation in the real motion of macromolecules.

Acknowledgment. Partial support by Bogazici University Research Funds Project 95P0056 and by the Turkish Academy of Sciences is gratefully acknowledged by B.E. and I.B.

\section{References and Notes}

(1) Baysal, C.; Atilgan, A. R.; Erman, B.; Bahar, I. J . Chem. Soc., Faraday Trans. 1995, 91, 2483.

(2) Bahar, I.; Erman, B.; Monnerie, L. Adv. Polym. Sci. 1994, $116,145$.

(3) J ones, A. A.; Stockmayer, W. H. J . Polym. Sci., Polym. Phys. Ed. 1977, 15, 847.

(4) Bendler, J. T.; Yaris, R. Macromolecules 1978, 11, 650.

(5) Hall, C.; Helfand, E. J . Chem. Phys. 1982, 77, 3275.

(6) Dejean de la Batie, R.; Laupretre, F.; Monnerie, L. Macromolecules 1989, 22, 122.

(7) Howarth, O. W. J . Chem. Soc., Faraday Trans. 2 1978, 74, 1031.

(8) Cook, R.; Helfand, E. J . Chem. Phys. 1985, 82, 1599.

(9) Moro, G. J . Chem. Phys. Lett. 1990, 173, 903.

(10) Kim, E.-G.; Mattice, W. L. J . Chem. Phys. 1994, 101, 6242.

(11) Bahar, I.; Erman, B. Macromolecules 1987, 20, 1369.

(12) Haliloglu, T.; Bahar, I.; Erman, B. J . Chem. Phys. 1992, 97, 4428.

(13) Baysal, C.; Erman, B.; Bahar, J . Macromolecules 1994, 27, 3650.

(14) Dauber-Osguthorpe, P.; Osguthorpe, D. J .J . Comput. Chem. 1993, 14, 1259.

(15) F or avoiding the redundant counting of isomeric transitions and eliminating the rotational jumps which are back-restored without stabilization of an isomeric state, the assignment of dihedral angles were performed using the ranges $\left|\varphi_{\mathrm{i}}\right|<30^{\circ}$ for the trans and $90^{\circ}<\left|\varphi_{i}\right|<135^{\circ}$ for gauche $( \pm)$ states. The rest of the dihedral angles are assigned their last definite states.

(16) Helfand, E.; Wasserman, Z. R.; Weber, T. A. J . Chem. Phys. 1981, 75, 4441.

MA9508818 